\title{
Challenges in Integration of Microelectronics \& Photonics with Nano-chemistry
}

\author{
Mohd. Muzaffar Ahmad ${ }^{1}$, Mohd. Abdul Sattar ${ }^{2}$, Mohd. Anas Ali \\ Professor, Dept of ECE, Nawab Shah Alam Khan College of Engineering \& Technology, Hyderabad, India ${ }^{1}$ \\ Associate Professor \& Head, Dept of ECE, Nawab Shah Alam Khan College of Engineering \& Technology, \\ Hyderabad, India ${ }^{2}$ \\ Low Voltage/Low Current Electronics Security System Engineer, Techno I Electronics Security System, \\ Hyderabad, India ${ }^{3}$
}

\begin{abstract}
Electronics, in particular VLSI technology, has made possible design of sophisticated, complex and powerful systems for user. Although silicon technology is evolving continuously to produce smaller, faster and reliable systems with low power, eventually the integration of Microelectronics and Photonics with Nano-chemistry as the driving force is the new area of research that spans from environmental management, smart health-care, smart energy management to new innovations in communications, processing and man-machine interfaces. This has created tremendous opportunities for realization of new systems. The co-existence of silicon technology with these emerging technologies, photonics and nano-chemistry has created new design opportunities for the designer, bringing new challenges to be solved. In this paper, some of the important challenges have been discussed.
\end{abstract}

Keywords: Photonics, nano-chemistry, VLSI Photonics, deep-submicron VLSI.

\section{INTRODUCTION}

Although silicon technology is in the process of processing techniques. Today, the telecommunication continuous evolution of producing smaller, faster \& community aims at Tera bits per second (Tbps) system reliable systems with low power, eventually the with a reach of $40 \mathrm{~km}$ routine.

integration of microelectronics with photonics and other Before, we talk of challenges arising because of emerging technologies is the driving force which has integration of technologies, let us talk of the challenges to created tremendous opportunities for realization of new be faced in deep-submicron VLSI.

systems. Photonics, specifically silicon photonics, is an The first challenge is interconnect. The delays through the alternative to electronics systems to transmit, distribute interconnect can easily be longer than the gate driving it. and process high volumes of digital information at a very Crosstalk between signals on wires can cause major fast speed. Other than its communication properties, the problems as well. Proper design methodologies and way light interacts/interfaces with other materials, makes careful analysis are keys to taming the problem introduced it an ideal tool for intelligent medical diagnosis and by the interconnect.

instrumentation of high precision and accuracy. The Another challenge is power consumption. Power integration of microelectronics with photon-based consumption is a concern on every large chip because of sciences, nano-chemistry and biotechnology brings into large amount of heat generated by so many transistors. forefront the future soc technology resulting into Excessive power consumption can make a chip so hot that intelligent Systems-on-Chip (isoc) products. These isocs it becomes unreliable and even non-functional. Careful will result in development of newer circuits and systems analysis of the power consumption is the remedy for with exra-ordinary new properties, almost in every keeping the power consumption within the specified segment of the economy. This integration lays down the limits.

foundation for a future in which sensing, imaging, Another challenge is reliability. As we move into information processing and communication can be nanometer-scale VLSI, the transistors become less integrated. Emerging concept of isoc in medical sciences reliable. We must apply reliability techniques at all levels will become the building block of an important tool kit for of abstraction - circuit, logic and architecture - if we are aging population. This evolution is the result of novel to cost effectively manage the transistors to nano-meter concepts that integrate microelectronics, photonics, nano- scale technology.

chemistry and biotechnology. CMOS photonics is aimed If we want to create complete systems-on-chip (socs), we at harnessing the massive bandwidth \& reach benefits of have to face the challenge of design complexity. Over the photonic technology, while enabling cost effective mass long run, the VLSI designers will have to become even manufacturing through use of established CMOS more skilled at programming, in addition to designing 
layout, circuit and architecture, as some fraction of the developments in Silicon Photonics, it is expected to bring system is implemented as on-chip software. A an optical revolution in Electronics \& Communication multiprocessor system-on-chip (mpsoc), has been industry with the realization of the above said Tbps data reported, which is a System-on-Chip with multiple links using micro-electronic silicon chips.

processing elements. Some of these mpsocs have regular architecture, while other mpsocs are heterogeneous.

\section{SILICON PHOTONICS}

Silicon Photonics can be defined as the utilization of silicon based materials for the generation, guidance, control and detection of light to communicate over distance. Optical technology suffered from a reputation as an expensive solution, based on high cost of hardware components, as they are typically fabricated using exotic materials that are expensive for manufacturing. These limitations prompted to research the construction of fiberoptic components from other materials such as silicon. Silicon photonics has attained much attention in recent years owing to the maturity of silicon in the electronics industry and its possibility of monolithic integration of both photonic and electronic devices on chip. It develops high volume low cost optical components using standard CMOS process - the IC manufacturing process used today.

Silicon photonics is an evolving technology in which data is transferred among computer chips by optical rays. Optical rays can carry far more data is less time than electrical conductors. Its overarching goal is to develop high volume, low cost optical components using standard CMOS processing the same manufacturing process used for microprocessors and semiconductor devices.

The components of silicon photonics are silicon light source, silicon wave of waveguide, silicon modulator, silicon based photo detector.

The work toward a silicon based light source is going on but still far from completion. Until an efficient reliable silicon based light source is available, a photonic integrated system will need to use a conventional III - V material light emitter. The basic silicon waveguide is a rib waveguide where as silicon rib is raised above a silicon base resting on an insulating layer; these waveguides are commonly referred to as SOI rib waveguides.

The silicon modulator is an external modulator, based on, based on the design of a Mach-Zender interferometer where an incoming beam of light is split in half and the two resulting beams travel through two different arms of the interferometer and then the two beams recombine at other end. The current design for a silicon based photo detector is to have a layer of $\mathrm{Si}-\mathrm{Ge}$ between a P-type and an N-type layer.

In the field of silicon photonics, there have been challenges in fabricating optical devices such as laser source, modulators, detectors etc. on silicon for high speed switching speeds that provide high data rates for communication links. Tbps data rate has already been achieved in optics with Dense wavelength Division Multiplexing (DWDM) technology. With further

\section{O-PCBS \& VLSI PHOTONICS}

The development of optical printed circuit boards (OPCBs) \& VLSI photonic circuits has been reported recently [1][2]. Micro/nano-photonic wires and devices are interconnected and integrated on a chip to form micro/nano circuits of various functions that are compact, high speed, intelligent, light weight, low energy and environment friendly, low cost and high volume applications. Photonic devices include micro/nano-scale lasers, switches, couplers, detectors, sensors, actuators, modulators and related devices to perform the functions of sensing, storing, transporting, processing, switching, routing and distributing optical signals on flat modulator boards or substrates for diverse applications. The O-PCBs are designed to overcome the limitations of the electrical PCBs and the VLSI photonic systems are designed to overcome the limitations of the VLSI electrical systems. The scientific \& technological challenges \& issues of interconnection and integration include diverse compatibility issues between micro/nano-devices of diverse nature, such as materials mismatch, size mismatch, shape mismatch, optical mismatch and mechanical/thermal mismatches. Coupling of the light from nano-wires $\mathrm{CdS}$ light emitting devices (LEDs) or nano-wire GaN LEDs into the photonic crystal nano-wave guides has been reported in the said \& related papers.

\section{SILICON PHOTONICS AND VLSI ELECTRONICS FOR COMPUTING SYSTEMS}

The work on computer systems based on silicon photonic interconnects has been reported [3]. The ever growing complexity, computation and communication requirements of military \& commercial applications continue to drive the need for scaling high performance computer systems. The architects have faced these challenges by not only increasing the clock speed, but also expanding the complexity of processor architectures, and devising chips with several multi-thread cores.

Processors with up to 8 cores \& 64 threads are now commercially available and commercial processors with much larger number of cores are in the offing. Designers have affectingly translated this increase in transistors into increase performance. Optical interconnected enables large collection of chips, giving high system performance, using optical proximity communication, allows, chips to communicate without a soldered connection and enables seamless re-workability for high system yield. WDM optics also offers unmatched bandwidth density. A microsystem architecture has been reported that takes advantage of new silicon photo interconnect technologies to create a collection of tightly connected chips that 
exhibit large bandwidths, balance communication and computation, and scalable improvement in performance.

A fundamental issue/challenge facing optical technology in the past has been the interface between the optical fiber $\&$ the die. This is exacerbated in the case of SOI waveguides since the silicon wave guide is extremely small, with a core only $0.1 \mathrm{um} 2$ in cross sectional area. By comparison, an optical fiber has a core with a cross sectional area $85 \mathrm{~nm} 2,3$ orders of magnitude larger. Thus coupling light from an optical fiber into a silicon waveguide will result into spillage and loss of the most of light around the edges.

Thus, it is important to focus the light from fiber down to the spot size of the silicon waveguide. this can be done efficiently by using a holographic lens. while it is quite obvious that low coupling loss is important in the performance of optical systems, the holographic lens also allows wafer scale test, both after the wafers are complete and during processing.

\section{VLSI AND NANO-CHEMISTRY / NANO TECHNOLOGY}

In early 1980's, scaling of the MOS transistors beyond $140 \mathrm{~nm}$ appeared as an impossible task. However, in subsequent years, manipulation of materials by nanochemists and material engineers, emergence of novel and copper technology and new techniques in lithography pave the way for realization of feature sizes of the order of 20 nm-30nm. Parallel with such development, new nanochemistry is trying to shape the new materials for the future. Better understanding of the unique properties of atoms and molecules, will result into progress of novel technologies that, in turn, will result into further development of VLSI circuits and system.

In a related development, there has been emergence of Carbon Nano Tubes (CNTs) which will revolutionize the development of electronic circuits and systems. Carbon Nano Tubes are hollow, cylindrical Nano - structures composed of single sheet of carbon atoms. They have exceptional electrical, physical and thermal properties. They are stronger than steel and 200 times lighter than steel. They are 1000 times thinner than human hair. Single Walled Carbon Nano Tubes (SWNT) have been used for building carbon nano tube computers. The structure of a SWNT can be visualized as a layer of graphite, a single atom thick called graphene, which is rolled into a seamless cylinder. Carbon Nano Tubes can exhibit both metal and semiconductor properties. Carbon Nano Tube Field effect transistors are promising candidate as an alternative of silicon CMOS. The CMOS VLSI, as we know it today, has certain limitations. There is huge amount of energy leakage. Also, we can't have feature size below $20 \mathrm{~nm}$. Quantum effect comes into action below $20 \mathrm{~nm}$, and therefore, we can't make transistors smaller than this. Digital circuits based on transistors fabricated from CNTs have the potential to outperform silicon by improving the energy delay product a metric of energy efficiency by more than an order of magnitude. Hence, CNTs are an exciting complement to existing semiconductor technologies.

At present, the processor speed is limited to $4 \mathrm{GHZ}$ per core. A group of scientists of Stanford university have created world's first carbon tube processor. This processor is basically free from energy leakage and is, thus, highly energy efficient. By using CNTs, we can create 10-15 GHZ. Processor in a single core. It can be implemented in present computer architectures like MIPS. The CNT computer runs an operating system that is capable of multi tasking. The future of carbon Nano Tube Computer lies in continuous improvement of computational power at low energy losses. Low metal usage helps to build environment friend processor.

\section{CONCLUSION}

In this paper we discussed some of the important challenges which arise due to integration of microelectronics and photonics, specifically VLSI photonics with nanochemistry. Some of these challenges have been solved while others are yet to be solved. For the challenges yet to be solved, some possible solutions have been suggested by us for a few of them.

\section{ACKNOWLEDGMENT}

We express our sincere gratitude to Prof. Mohd. Muzaffar Ahmad, Electronics \& Communication Engineering Department, Nawab Shah Alam Khan College of Engineering \& Technology, Hyderabad, for extending his valuable insight for completion this work.

\section{REFERENCES}

[1] E. H. Lee "Optical Circuit Board (O-PCB) as a platform for VLSI Photonics”, IEEE/LEDs, Summer Topical Meeting. San Diego, CA, USA, June 29-34, 2004.

[2] E. H. Lee "Micro/Nano-Photonic Integration on Optical Printed Circuit Board (O-PCB) “ SPIE- Europe, Strasbourg, France, April 3-7, 2006.

[3] Manish, S Barreh, J., Brooks, J., Golla, R., Grohski, G, Gura, Bikram, S., Sheam, D., Sparcklen, L., Wynn, A. "Ultra SPARC T2: A highly threaded, power-efficient, SPARC SOC, IEEE asian solid State Circuits Conference (ASSCC' 07), 12-14 Nov 2007, pp 2225, 2007.

[4] A. V. Krishnamoorthy and D.A.B. Miller "firehose architectures for free space optically interconnected VLSI circuits", J. Parallel and Distributed Computing, vol 41, pp 109-114, 1997.

[5] A. V. Krishnamoorthy and D.A.B. Miller "Scaling optoelectronic VLSI circuits into the $21^{\text {st }}$ century: A technology roadmap", IEEEJ Select Topics Quantum Electron, vol 2, pp 55-76, Apr 1996.

[6] D. Zhon, F. P. Preparata, and S.M. Kaug, "Interconnection delay in very speed VLSI", IEEE Trans. Circuits Syst. Vol 38, pp 779-789 July 1991.

[7] R. A. Soref and BR Bennet, "Electro optical effects in Silicon" IEEEJ of quantum Electron, vol QE - 23, No. 1, Jan 1987, pp 123-9.

[8] R. Chemberlain, M. Franklin, R. Krchnavek and B. Baysal, "Design of an optically-interconnected multicomputer" Proc. Of $5^{\text {th }}$ Int'l conf. on Massively Parallel Processing using Optical interconnects, pp 114-122, June 1998. 
[9] R. G. Beausoleil, "Large scale integrated photonics for high performance interconnects", J. Emerg. Technol. Comput. Syst. 7, 154 (2011).

[10] K. Dhira, K. Kobayashi, N. lizuka, Hyoshida, M. Ezaki, H. Uemura, A Kojima, K. Nakamura, H. Furuyama, H. Shibata " Onchip optical interconnection by using integrated III-IV Laser diode and photodetector with silicon waveguide", Opt. Express 18, 15440-15447 (2010).

\section{BIOGRAPHIES}

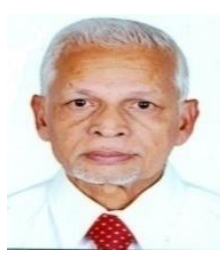

Mohammad Muzaffar Ahmad, received B. Tech. Degree in Electrical Engineering from Indian Institute of Technology (IIT), Kanpur and M.Tech. in Digital \& Computing Systems from Indian Institute of Technology(IIT), Kanpur . He was a Professor \& Head of the Dept. of ECE in Nawab Shah Alam Khan College of Engineering \& Technology, Malakpet, Hyderabad. He is also a retired scientist of DRDO

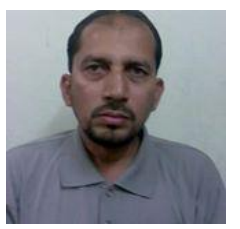

Mohd Abdul Sattar, received B.Tech. Degree in Electronics and Communication Engineering from National Institute of Technology(NIT), Warangal and M.Tech. in Embedded Systems from JNTUH. $\mathrm{He}$ is an Associate Professor \& Head of the Dept. of ECE in Nawab Shah Alam Khan College of Engineering \& Technology, Malakpet, Hyderabad. He is also a member of IEEE.

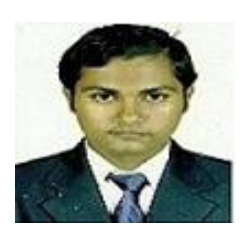

Mohd Anas Ali received B.Tech. Degree in Electronics and Communication Engineering from Pujya Shri Madhavanji College of Engineering \& Technology affiliated to JNTU Hyderabad in 2013 \& M.Tech. degree in Embedded System from Nawab Shah Alam Khan College of Engineering \& Technology. He is presently working at Techno I Security Systems, Abids, Hyderabad. 